\title{
EVOLUCIÓN A CORTO PLAZO DE LAS PROPIEDADES QUÍMICAS EN DOS SUELOS DE LA ZONA CENTRO SUR DE CHILE FERTILIZADOS CON DIFERENTES FUENTES DE CALCIO
}

\section{SHORT-TERM EVOLUTION OF THE CHEMICAL PROPERTIES OF TWO SOILS FROM SOUTH-CENTRAL CHILE FERTILIZED WITH DIFFERENT CALCIUM SOURCES}

\author{
Juan Hirzel $^{1 *}$, Sonia Toloza ${ }^{2}$, y Franco Novoa ${ }^{2}$ \\ ${ }^{1}$ Instituto de Investigaciones Agropecuarias, Centro Regional de Investigación Quilamapu, Avda. \\ Vicente Méndez 515, Chillán, Chile. \\ ${ }^{2}$ Empresa Ibiterra Chile Ltda, Pudahuel Poniente 1092, Santiago, Chile. \\ *Autor para correspondencia E-mail: jhirzel@inia.cl
}

\section{RESUMEN}

El uso de fertilizantes cálcicos genera cambios en las propiedades químicas del suelo, que a mediano y largo plazo afectan principalmente la acidez, suma de bases (SB), y concentraciones de calcio (Ca) y magnesio $(\mathrm{Mg})$. Sin embargo, no hay información sobre cambios en el corto plazo, por lo cual se realizó un estudio en condiciones controladas con dos suelos de la zona centro-sur de Chile, clases texturales franca (Haplaquept) y arcillosa (Duraqualf), respectivamente, empleando diferentes fuentes de cálcio (Ibisoil, Nitrato, Cloruro, Sulfato, y Carbonato de Ca), con el objetivo de determinar la fuente cálcica de disponibilidad más rápida y mejor impacto sobre las propiedades químicas. Todos los suelos fueron fertilizados con una dosis de $100 \mathrm{mg} \mathrm{kg}^{-1}$ de Ca e incubados por 16 días a $25^{\circ} \mathrm{C} \mathrm{y} 80 \%$ de humedad aprovechable. Los resultados indicaron diferencias en algunas propiedades químicas dependientes del tipo de suelo. El mayor pH se logró con el uso de Carbonato de Ca sólo en el suelo franco, sin detectar diferencias en el suelo arcilloso. En ambos suelos la concentración de Ca y potasio no fue afectada por los tratamientos de fertilización, pero todos presentaron aumento de Ca en relación al testigo. La mayor relación de $\mathrm{Ca} / \mathrm{SB}$ se logró con Ibisoil, la menor conductividad eléctrica (CE) en ambos suelos se logró con Ibisoil y Carbonato de Ca, y la mayor concentración de sodio y CE se obtuvo con Cloruro de Ca. Finalmente, el fertilizante con rápido incremento en la relación $\mathrm{Ca} / \mathrm{SB}$ y menor CE fue Ibisoil.

Palabras clave: calcio, fertilizantes, propiedades químicas, suelo, cambios de corto plazo.

\section{ABSTRACT}

The use of fertilizers based on calcium (Ca) generates changes in the soil chemical properties, affecting acidity, sum of bases (BS), and $\mathrm{Ca}$ and magnesium $(\mathrm{Mg})$ concentrations in the medium to long term. However, there is no information available about short-term changes. A study was conducted to determinate the most readily available Ca source and the best impact on the chemical properties of two different types of soils from south-central Chile, texture class loamy (Haplaquept) and clay (Duraqualf), respectively. The study was conducted under controlled conditions, using different Ca sources (Ibisoil, Nitrate, Clorhide, Sulphate, and Carbonate of Ca). The soils were fertilized with $100 \mathrm{mg} \mathrm{kg}^{-1}$ of $\mathrm{Ca}$ and incubated for 16 days at $25^{\circ} \mathrm{C}$ and $80 \%$ of available water. Results 
indicate differences in some chemical properties that depend on the soil. The highest $\mathrm{pH}$ value was obtained with the use of carbonate alone in the loam soil, while no differences were found in the clay soil. Similarly, no significant differences were observed in terms of $\mathrm{Ca}$ and $\mathrm{K}$ concentrations due to the fertilization treatments in any of the soils, but all of them increased Ca concentration compared with the control treatment. The highest $\mathrm{Ca} / \mathrm{BS}$ ratio was obtained with Ibisoil. The lowest electric conductivity (EC) was obtained with Ibisoil and Ca Carbonate in both soils, while the highest sodium concentration and EC values were obtained with Ca Clorhide. The fertilizer with the highest increase in the $\mathrm{Ca} / \mathrm{BS}$ ratio and the lowest $\mathrm{EC}$ was Ibisoil.

Key words: calcium, fertilizers, chemical properties, soil, short-time changes.

\section{INTRODUCCIÓN}

La fertilidad del suelo, entendida como la capacidad para producir bienes y servicios en forma sustentable, es un concepto amplio que involucra propiedades físicas, químicas y biológicas (Havlin et al., 1999; Baldi et al., 2006), sin embargo, el uso continuo del suelo sin realización de prácticas de conservación ha generado una pérdida paulatina de la calidad de estas propiedades. Dentro de las propiedades químicas, la acidez del suelo, asociada con las concentraciones de aluminio $(\mathrm{Al})$ e hidrógeno $(\mathrm{H})$, y la disponibilidad de bases de intercambio como calcio $(\mathrm{Ca})$, magnesio $(\mathrm{Mg})$, potasio $(\mathrm{K})$ y sodio $(\mathrm{Na})$, que contrarrestan esta acidez, son reportadas como un componente importante de la fertilidad del suelo (Castro y Crusciol, 2013; Fageria y Nascente; 2014; Fageria et al., 2014; Moreira et al., 2015). Otros componentes de importancia en las propiedades químicas del suelo son también los nutrientes esenciales fósforo $(\mathrm{P})$, nitrógeno $(\mathrm{N})$, azufre $(\mathrm{S})$ y micronutrientes (Hirzel et al., 2007; Hirzel et al., 2010; Castro y Crusciol, 2013).

El aumento en la concentración de bases disponibles en el suelo, producto del encalado o del uso de fertilizantes cálcicos, permite reducir la disponibilidad de $\mathrm{Al}$ e $\mathrm{H}$, y con ello reducir la acidez e incrementar el $\mathrm{pH}$ del suelo (Castro and Crusciol, 2013; Fageria and Nascente; 2014; Caires et al., 2015; Moreira et al., 2015). A su vez, estos cambios producidos por el incremento en la concentración de bases en el suelo permiten aumentar la disponibilidad de algunos nutrientes esenciales y también aumentar la productividad de la mayoría de las plantas cultivadas (Castro and Crusciol, 2013; Fageria and Nascente; 2014; Moreira et al., 2015). Por ello, el uso de enmiendas calcáreas y de fertilizantes cálcicos es cada vez más común en la agricultura, con ventajas de disponibilidad en el caso de fertilizantes cálcicos (Fageria et al., 2014; Moore and Ouimet, 2014), y de menor costo en el caso de enmiendas calcáreas.

Si bien los estudios de uso de enmiendas calcáreas y fertilizantes cálcicos reportan resultados positivos de productividad en plantas indicadoras, con efectos a mediano y largo plazo (uno o más años) sobre las propiedades del suelo (Castro and Crusciol, 2013; Fageria and Nascente, 2014; Caires et al., 2015; Moreira et al., 2015), no se mencionan efectos de inmediato y corto plazo (pocos días o pocos meses después de aplicadas las fuentes de $\mathrm{Ca}$ ) que evalúen las diferentes alternativas de fertilizantes cálcicos existentes. Los efectos de corto plazo que contribuyan a una mayor disponibilidad de calcio y mejor condición química y nutricional, están asociados a un mayor crecimiento inicial de raíces (Maathuis, 2009; Marschner, 2012), lo cual puede generar un mejor establecimiento del cultivo y mayor rendimiento potencial. Por otra parte, dentro de los fertilizantes cálcicos disponibles, el anión acompañante al calcio puede ser de diferente origen, correspondiendo principalmente a compuestos inorgánicos como Nitratos, Cloruros, Sulfatos y Carbonatos (Román y Gutiérrez, 1998; Castro y Crusciol, 2013; Fageria y Nascente; 2014; Moore y Ouimet, 2014; Moreira et al., 2015), y compuestos orgánicos como los ácidos carboxílicos (Román and Gutiérrez, 1998; Flores et al., 2014), con un mejor efecto de productividad cuando se emplean estos últimos (Román and Gutiérrez, 1998; Flores et al., 2014).

El objetivo de este estudio fue determinar la evolución durante los primeros 16 días (considerado como corto plazo) y en condiciones controladas del $\mathrm{pH}$, conductividad eléctrica (CE), y concentraciones de $\mathrm{Ca}, \mathrm{Mg}$, $\mathrm{K}$ y $\mathrm{Na}$ en dos suelos de distinta textura de la zona centro sur de Chile, fertilizados con las principales fuentes cálcicas disponibles en el país. A su vez, se buscó determinar la fuente cálcica de disponibilidad más rápida y de mejor impacto sobre las propiedades químicas evaluadas.

\section{MATERIALES Y MÉTODOS}

Los suelos empleados en el experimento fueron colectados a una profundidad de 0 a $20 \mathrm{~cm}$, desde dos huertos de manzano (Malus doméstica Borkh.) de la zona de Molina, región del Maule. El primer suelo, localizado en las coordenadas $35^{\circ} 05^{\prime} 17,31^{\prime \prime} \mathrm{S}, 71^{\circ} 15^{\prime} 48,78^{\prime \prime} \mathrm{O}$, con texturas franco (Haplaquepts) y el otro suelo, ubicado en 
Tabla 1. Propiedades físico-químicas de los suelos $(0-20 \mathrm{~cm})$ previo al inicio del experimento. Table 1. Physico-chemical properties of the soils $(0-20 \mathrm{~cm})$ prior to the start of the experiment.

\begin{tabular}{lcc}
\hline Parámetro & Suelo franco & Suelo arcilloso \\
\hline Arcilla, \% & 17,9 & 41,3 \\
Limo, \% & 31,6 & 26,7 \\
Arena, \% & 50,5 & 32,0 \\
Humedad gravimétrica a 0,33 bares & 23,2 & 25,2 \\
Humedad gravimétrica a 15,00 bares & 11,4 & 16,4 \\
pH (suelo:agua 1:5) & 6,66 & 5,61 \\
Materia orgánica, $\mathrm{g} \mathrm{kg}^{-1}$ & 3,61 & 3,65 \\
Conductividad eléctrica, dS m ${ }^{-1}$ & 0,007 & 0,008 \\
N disponible, mg kg-1 & 5,0 & 42,0 \\
P Olsen, mg kg-1 & 21,0 & 22,2 \\
K intercambiable, cmol+ kg-1 & 0,46 & 0,44 \\
Ca intercambiable, cmol+ kg-1 & 7,86 & 5,10 \\
Mg intercambiable, cmol+ kg-1 & 1,60 & 1,48 \\
Na intercambiable, cmol+ $\mathrm{kg}^{-1}$ & 0,21 & 0,07 \\
Al intercambiable, cmol+ kg-1 & 0,03 & 0,05 \\
Fe disponible, mg kg-1 & 126,0 & 53,0 \\
Mn disponible, mg kg-1 & 6,1 & 45,7 \\
Zn disponible, mg kg-1 & 3,2 & 2,1 \\
Cu disponible, mg kg-1 & 9,2 & 6,9 \\
B disponible, mg kg-1 & 1,1 & 0,7 \\
S disponible, mg kg-1 & 2,0 & 13,3 \\
\hline
\end{tabular}

las coordenadas $35^{\circ} 11^{\prime} 17,08^{\prime \prime} \mathrm{S}, 71^{\circ} 16^{\prime} 45^{\prime \prime} \mathrm{O}$, con textura arcillosa (Duraqualfs) (USDA, 2014), cuyas propiedades físico-químicas se presentan en el Tabla 1. La determinación de las propiedades químicas fue realizada de acuerdo a la metodología indicada por la Comisión Nacional de Acreditación de Análisis de Suelo (Sadzawka et al., 2006). La humedad del suelo fue determinada por gravimetría, expresando los resultados en base a peso seco. La textura del suelo fue determinada a través del método del hidrómetro de Bouyoucos (Burt, 2004) (Tabla 1).

El experimento se desarrolló en condiciones aeróbicas controladas, con incubación de suelos a $25^{\circ} \mathrm{C}$ y $80 \%$ de la humedad aprovechable, como una modificación del método señalado por Laos et al. (2000). Se emplearon frascos plásticos de $250 \mathrm{~cm}^{3}$ de capacidad, a los cuales se les agregó $100 \mathrm{~g}$ de suelo seco, tamizado a $2 \mathrm{~mm}$, y los tratamientos de fertilización cálcica, con reposición de humedad cada 2 días para recuperar la humedad inicial, usando agua destilada. Los tiempos de incubación fueron de 1, 2, 4, 8 y 16 días, considerando también un tiempo de 0 días como condición de inicio del experimento. Se empleó una cámara de incubación (FOC 225E Refrigerated Incubator Velp Scientifica, New York, USA).

Los tratamientos evaluados fueron: 1) control sin fertilización; 2) Ibisoil Ca® (Hidróxido de Ca acomplejado con los ácidos carboxílicos succínico y málico, 35\% $\mathrm{CaO}$, en estado de fluido viscoso) (Ibisoil); 3) Nitrato de $\mathrm{Ca}(26 \% \mathrm{CaO})$ en estado de polvo fino con malla menor a 40 mesh; 4) Cloruro de $\mathrm{Ca}(38 \%$ de $\mathrm{CaO})$ en estado de polvo fino con malla menor a 20 mesh; 5) Sulfato de Ca (32,5\% $\mathrm{CaO}$ ) en estado de polvo con malla menor a 200 mesh; y 6) Carbonato de $\mathrm{Ca}(56 \%$ de $\mathrm{CaO}$ ) en estado de polvo fino con malla menor a 38 mesh, en una dosis de $100 \mathrm{mg} \mathrm{kg}^{-1}$ de Ca. Todos los fertilizantes, excepto Ibisoil de presentación fluida, fueron molidos en mortero para facilitar el establecimiento de los experimentos, dada la baja dosis a emplear de cada producto para lograr la concentración de $100 \mathrm{mg} \mathrm{kg}^{-1}$ de Ca. Esta molienda de los fertilizantes permitió aplicar pequeñas cantidades de cada fuente cálcica en la masa de suelo utilizada en cada pote, y además mejorar su solubilidad, dado el menor tamaño de partícula y la mayor superficie de contacto con el agua del suelo. Al mejorar la solubilidad de cada fuente cálcica sólida como efecto del menor tamaño de partícula obtenido, se mejora su capacidad de reaccionar sobre las propiedades químicas del suelo en el corto plazo, y de esta forma acceder a los efectos buscados con este experimento (cambios de corto plazo). Cada tratamiento contó con 3 réplicas. Todas las fuentes de Ca se aplicaron en polvo, excepto Ibisoil que fue aplicado disuelto 
en agua destilada en relación 1:10.

El diseño experimental fue de parcelas sub-divididas, donde la parcela principal fue el suelo, la sub-parcela el tiempo de incubación, y la sub-sub-parcela el tratamiento de fertilización cálcica. Los resultados considerados como una población normalizada fueron sometidos a análisis de varianza, y las diferencias de medias fueron analizadas con el Test de Tukey para un 5\% de significancia. A su vez, las interacciones fueron separadas a través de análisis de contrastes, para los promedios obtenidos del periodo de incubación. Para todos estos estos análisis se empleó el software SAS System versión 6.0 (SAS, 1989). Las evaluaciones realizadas fueron $\mathrm{pH}$ al agua, conductividad eléctrica (CE), concentraciones de bases $\mathrm{Ca}, \mathrm{Mg}, \mathrm{K}, \mathrm{y} \mathrm{Na}$, a través de la metodología indicada por Sadzawka et al. (2006), y la suma de bases (SB) como una determinación matemática de las bases $\mathrm{Ca}, \mathrm{Mg}$, $\mathrm{K}$ y $\mathrm{Na}$. A su vez la relación $\mathrm{Ca} / \mathrm{SB}$ se calculó como una relación matemática entre ambos parámetros.

\section{RESULTADOS Y DISCUSION}

Los suelos empleados en el experimento presentan niveles medios a altos de Ca (Tabla 1). Sin embargo, la disponibilidad real de Ca depende de las tasas de desorción de este elemento, las cuales son bajas en relación a las otras bases presentes (Havlin et al. 1999), y muchas veces insuficientes para lograr atributos de calidad y condición en especies cuyo comportamiento de postcosecha tiene mucho valor comercial. Al respecto, Tagliavini et al. (2000) y Zavalloni et al. (2001), señalan que uno de los factores que permite incrementar el consumo de Ca por una planta es la alta disponibilidad de este elemento en la solución del suelo, situación que mejora con la fertilización cálcica.

El análisis estadístico (Tabla 2), indicó que to- das las variables evaluadas $(\mathrm{pH}, \mathrm{CE}, \mathrm{Ca}, \mathrm{Mg}, \mathrm{K}$, $\mathrm{Na}$ y relación $\mathrm{Ca} / \mathrm{SB}$ ) fueron afectados tanto por el suelo como por el tratamiento de fertilización. Sin embargo, el tiempo de incubación no tuvo efecto sobre el $\mathrm{pH}$ del suelo (Tabla 2). A su vez, los parámetros $\mathrm{Ca}, \mathrm{Mg}$, $\mathrm{Na}$ y relación $\mathrm{Ca} / \mathrm{SB}$ fueron afectados por las interacciones suelo $\mathrm{x}$ tiempo de incubación, y suelo $x$ tratamiento de fertilización cálcica (Tabla 2). Por su parte, la triple interacción suelo $\mathrm{x}$ tiempo de incubación $\mathrm{x}$ tratamiento de fertilización cálcica, afectó los parámetros $\mathrm{CE}, \mathrm{Ca}$, $\mathrm{Mg}$, Na y relación Ca/SB (Tabla 2).

Dadas las interacciones obtenidas entre las variables evaluadas (Tabla 2), se contrastó el efecto del suelo y el efecto de los tiempos de incubación sobre las propiedades analizadas en ambos suelos (Tablas 3 y 4, respectivamente). A su vez, como el efecto del suelo sobre las propiedades químicas fue significativamente muy evidente (Tablas $2 \mathrm{y}$ 3) y generó interacción con los tratamientos fertilizantes para la mayoría de las propiedades evaluadas (Tabla 2), los efectos de los tratamientos fertilizantes se presentan separados por tipo de suelo (Tablas 5 y 6, para el suelo franco y arcilloso, respectivamente). Por su parte, la evolución en el tiempo de las propiedades químicas se presenta en la Fig. 1, para el suelo franco, y en la Fig. 2 para el suelo arcilloso.

\section{Efecto del suelo}

Respecto al efecto del suelo, y como promedio de todo el periodo de evaluación y de los tratamientos de fertilización cálcica (Tabla 3), se esperaban diferencias asociadas al contenido de arcillas de cada uno, dado que este parámetro afecta la capacidad de intercambio catiónico (CIC) y con ello las relaciones de adsorción y desorción de bases (Havlin et al. 1999; Fageria y Nascente, 2014). El suelo franco presentó mayores valores en todas las variables $(\mathrm{p}<0,05)$, excepto en la CE (Tabla $3)$, asociado al mayor valor inicial de las propie-

Tabla 2. Niveles de significancia para los parámetros evaluados en el experimento.

Table 2. Significance levels for the parameters evaluated in the experiment.

\begin{tabular}{lcccccc}
\hline Parámetro & $\begin{array}{c}\text { Suelo } \\
\text { (S) }\end{array}$ & $\begin{array}{c}\text { Tiempo incubación } \\
\text { (T) }\end{array}$ & $\begin{array}{c}\text { Tratamiento } \\
\text { (F) }\end{array}$ & $\begin{array}{c}\text { Interacción } \\
S^{*} \mathrm{~T}\end{array}$ & $\begin{array}{c}\text { Interacción } \\
S^{*} \mathrm{~F}\end{array}$ & $\begin{array}{c}\text { Interacción } \\
\mathrm{S}^{*} \mathrm{~T}^{*} \mathrm{~F}\end{array}$ \\
\hline $\mathrm{pH}$ & $* *$ & $\mathrm{~ns}$ & $* *$ & $\mathrm{~ns}$ & $\mathrm{~ns}$ & $\mathrm{~ns}$ \\
$\mathrm{CE}$ & $* *$ & $* *$ & $* *$ & $\mathrm{~ns}$ & $\mathrm{~ns}$ & $* *$ \\
$\mathrm{Ca}$ & $* *$ & $* *$ & $* *$ & $* *$ & $* *$ & $* *$ \\
$\mathrm{Mg}$ & $* *$ & $* *$ & $* *$ & $* *$ & $* *$ & $* *$ \\
$\mathrm{~K}$ & $* *$ & $* *$ & $* *$ & $\mathrm{~ns}$ & $\mathrm{~ns}$ & $\mathrm{~ns}$ \\
$\mathrm{Na}$ & $* *$ & $* *$ & $* *$ & $* *$ & $* *$ & $* *$ \\
$\mathrm{Ca} / \mathrm{SB}^{+}$ & $* *$ & $* *$ & $* *$ & $* *$ & $* *$ & $*$ \\
\hline
\end{tabular}

${ }^{+} \mathrm{SB}$, suma de bases $(\mathrm{Ca}+\mathrm{Mg}+\mathrm{K}+\mathrm{Na})$.

* Significativo a $\mathrm{P}<0,05$; ${ }^{* *}$ Significativo a $\mathrm{P}<0,01$; ns, no significativo 
Tabla 3. Análisis de contrastes para separación entre suelos para el valor promedio del periodo y de los tratamientos de fertilización cálcica.

Table 3. Contrast analysis between soils for the average value of the period and the calcium fertilization treatments.

\begin{tabular}{lcc}
\hline Parámetro & Suelo franco & Suelo arcilloso \\
\hline $\mathrm{pH}$ (suelo:agua 1:2,5) & $6,48 \mathrm{a}$ & $5,40 \mathrm{~b}$ \\
$\mathrm{CE}, \mathrm{dS} \mathrm{m}{ }^{-1}$ & $0,0097 \mathrm{~b}$ & $0,0132 \mathrm{a}$ \\
$\mathrm{Ca}, \mathrm{cmol}^{-\mathrm{kg}^{-1}}$ & $8,00 \mathrm{a}$ & $5,19 \mathrm{~b}$ \\
$\mathrm{Mg}, \mathrm{cmol}+\mathrm{kg}^{-1}$ & $1,53 \mathrm{a}$ & $1,37 \mathrm{~b}$ \\
$\mathrm{~K}, \mathrm{cmol}+\mathrm{kg}^{-1}$ & $0,459 \mathrm{a}$ & $0,437 \mathrm{~b}$ \\
$\mathrm{Na}, \mathrm{cmol}+\mathrm{kg}^{-1}$ & $0,217 \mathrm{a}$ & $0,079 \mathrm{~b}$ \\
$\mathrm{Ca} / \mathrm{SB}^{+}(\%)$ & $78,4 \mathrm{a}$ & $73,3 \mathrm{~b}$ \\
\hline
\end{tabular}

${ }^{\dagger} \mathrm{SB}$, suma de bases $(\mathrm{Ca}+\mathrm{Mg}+\mathrm{K}+\mathrm{Na})$.

Letras distintas en una misma fila indican diferencia estadística entre medias según test de Tukey $(p<0,05)$

Tabla 4. Análisis de contrastes para separación entre tiempos de incubación para el promedio de ambos suelos y de los tratamientos de fertilización cálcica.

Table 4. Contrast analysis between incubation times for the average value of both soils and the calcium fertilization treatments.

\begin{tabular}{lcccccc}
\hline Parámetro & \multicolumn{7}{c}{ Tiempo de incubación (días) } \\
& $\mathbf{0}$ & $\mathbf{1}$ & $\mathbf{2}$ & $\mathbf{4}$ & $\mathbf{8}$ & $\mathbf{1 6}$ \\
\hline $\mathrm{pH}($ suelo:agua 1:2,5) & $5,83 \mathrm{a}$ & $5,98 \mathrm{a}$ & $6,02 \mathrm{a}$ & $5,96 \mathrm{a}$ & $5,97 \mathrm{a}$ & $5,87 \mathrm{a}$ \\
$\mathrm{CE}, \mathrm{dS} \mathrm{m} \mathrm{m}^{-1}$ & $0,0111 \mathrm{a}$ & $0,0112 \mathrm{a}$ & $0,0112 \mathrm{a}$ & $0,0116 \mathrm{a}$ & $0,012 \mathrm{a}$ & $0,012 \mathrm{a}$ \\
$\mathrm{Ca}, \mathrm{cmol}^{-1} \mathrm{~kg}^{-1}$ & $6,29 \mathrm{a}$ & $6,46 \mathrm{a}$ & $6,52 \mathrm{a}$ & $6,81 \mathrm{a}$ & $6,83 \mathrm{a}$ & $6,66 \mathrm{a}$ \\
$\mathrm{Mg}, \mathrm{cmol}^{-1} \mathrm{~kg}^{-1}$ & $1,39 \mathrm{~b}$ & $1,42 \mathrm{ab}$ & $1,50 \mathrm{ab}$ & $1,43 \mathrm{ab}$ & $1,42 \mathrm{ab}$ & $1,53 \mathrm{a}$ \\
$\mathrm{K}, \mathrm{cmol}^{-1} \mathrm{~kg}^{-1}$ & $0,434 \mathrm{a}$ & $0,455 \mathrm{a}$ & $0,446 \mathrm{a}$ & $0,461 \mathrm{a}$ & $0,456 \mathrm{a}$ & $0,437 \mathrm{a}$ \\
$\mathrm{Na}, \mathrm{cmol}^{-1} \mathrm{~kg}^{-1}$ & $0,131 \mathrm{c}$ & $0,138 \mathrm{bc}$ & $0,141 \mathrm{bc}$ & $0,154 \mathrm{ab}$ & $0,154 \mathrm{ab}$ & $0,168 \mathrm{a}$ \\
$\mathrm{Ca} / \mathrm{SB}^{+}(\%)$ & $75,8 \mathrm{ab}$ & $75,8 \mathrm{ab}$ & $75,2 \mathrm{ab}$ & $76,5 \mathrm{ab}$ & $76,8 \mathrm{a}$ & $75,0 \mathrm{~b}$
\end{tabular}

${ }^{+} \mathrm{SB}$, suma de bases $(\mathrm{Ca}+\mathrm{Mg}+\mathrm{K}+\mathrm{Na})$.

Letras distintas en una misma fila indican diferencia estadística entre medias según test de Tukey $(\mathrm{p}<0,05)$

Tabla 5. Análisis de contrastes para separación entre tratamientos de fertilización en el suelo franco para el promedio del periodo de evaluación.

Table 5. Contrast analysis between fertilization treatments in the loam soil for the average value of the evaluation period.

\begin{tabular}{|c|c|c|c|c|c|c|}
\hline \multirow[t]{2}{*}{ Parámetro } & \multicolumn{6}{|c|}{ Tratamientos de fertilizantes cálcicos } \\
\hline & Control & Ibisoil & Nitrato & Cloruro & Sulfato & Carbonato \\
\hline pH (suelo:agua 1:2,5) & $6,56 \mathrm{c}$ & $6,72 \mathrm{~b}$ & $6,35 \mathrm{~d}$ & $6,24 \mathrm{f}$ & $6,31 \mathrm{e}$ & $6,74 \mathrm{a}$ \\
\hline $\mathrm{CE}, \mathrm{dS} \mathrm{m}^{-1}$ & $0,00522 \mathrm{~d}$ & $0,00534 \mathrm{~d}$ & $0,01114 \mathrm{c}$ & $0,01644 \mathrm{a}$ & $0,01439 \mathrm{~b}$ & $0,00594 \mathrm{~d}$ \\
\hline $\mathrm{Ca}, \mathrm{cmol}+\mathrm{kg}^{-1}$ & $7,49 \mathrm{~b}$ & $8,01 \mathrm{a}$ & $8,01 \mathrm{a}$ & 8,14 a & $8,32 \mathrm{a}$ & 8,05 a \\
\hline $\mathrm{Mg}$, cmol+ $\mathrm{kg}^{-1}$ & $1,51 \mathrm{ab}$ & $1, .49 \mathrm{~b}$ & $1,55 \mathrm{ab}$ & $1,54 \mathrm{ab}$ & $1,56 \mathrm{a}$ & $1,53 \mathrm{ab}$ \\
\hline $\mathrm{K}, \mathrm{cmol}+\mathrm{kg}^{-1}$ & 0,449 a & $0,452 \mathrm{a}$ & 0,461 a & 0,469 a & $0,465 \mathrm{a}$ & 0,458 a \\
\hline $\mathrm{Na}, \mathrm{cmol}+\mathrm{kg}^{-1}$ & $0,208 \mathrm{~b}$ & $0,214 b$ & $0,218 \mathrm{ab}$ & $0,229 a$ & $0,216 b$ & $0,214 b$ \\
\hline $\mathrm{Ca} / \mathrm{SB}^{+}(\%)$ & $77,6 \mathrm{c}$ & $78,8 \mathrm{a}$ & $78,2 \mathrm{~b}$ & $78,5 \mathrm{ab}$ & $78,8 \mathrm{a}$ & $78,5 \mathrm{ab}$ \\
\hline
\end{tabular}

${ }^{+} \mathrm{SB}$, suma de bases $(\mathrm{Ca}+\mathrm{Mg}+\mathrm{K}+\mathrm{Na})$.

Letras distintas en una misma fila indican diferencia estadística entre medias según test de Tukey $(p<0,05)$

dades analizadas (Tabla 1), y en el caso de la CE, debido al menor contenido de arcillas (Havlin et al. 1999) y en menor medida al valor inicial del suelo (Tabla 1). La CE está asociada a la fracción soluble en agua, la cual no fue medida en este experimento, y al contenido de partículas finas (Hossain et al., 2015). Por su parte, un suelo con mayor nivel de disponibilidad de nutrientes des- 
Tabla 6. Análisis de contrastes para separación entre tratamientos de fertilización en el suelo arcilloso para el promedio del periodo de evaluación.

Table 6. Contrast analysis between fertilization treatments in the clay soil for the average value of the evaluation period.

\begin{tabular}{lllllll}
\hline Parámetro & \multicolumn{6}{c}{ Tratamientos de fertilizantes cálcicos } \\
& Control & Ibisoil & Nitrato & Cloruro & Sulfato & Carbonato \\
\hline $\mathrm{pH}$ (suelo:agua 1:2,5) & $5,46 \mathrm{a}$ & $5,35 \mathrm{a}$ & $5,30 \mathrm{a}$ & $5,25 \mathrm{a}$ & $5,38 \mathrm{a}$ & $5,63 \mathrm{a}$ \\
$\mathrm{CE}, \mathrm{dS} \mathrm{m}{ }^{-1}$ & $0,00882 \mathrm{e}$ & $0,00966 \mathrm{~d}$ & $0,01711 \mathrm{~b}$ & $0,02039 \mathrm{a}$ & $0,01467 \mathrm{c}$ & $0,00901 \mathrm{de}$ \\
$\mathrm{Ca}, \mathrm{cmol}^{+} \mathrm{kg}^{-1}$ & $4,78 \mathrm{~b}$ & $5,30 \mathrm{a}$ & $5,20 \mathrm{a}$ & $5,30 \mathrm{a}$ & $5,31 \mathrm{a}$ & $5,26 \mathrm{a}$ \\
$\mathrm{Mg}, \mathrm{cmol}^{-1} \mathrm{~kg}^{-1}$ & $1,35 \mathrm{~b}$ & $1,36 \mathrm{ab}$ & $1,39 \mathrm{ab}$ & $1,40 \mathrm{a}$ & $1,37 \mathrm{ab}$ & $1,35 \mathrm{~b}$ \\
$\mathrm{~K}, \mathrm{cmol}^{-1} \mathrm{~kg}^{-1}$ & $0,426 \mathrm{~b}$ & $0,435 \mathrm{ab}$ & $0,445 \mathrm{a}$ & $0,445 \mathrm{a}$ & $0,437 \mathrm{ab}$ & $0,436 \mathrm{ab}$ \\
$\mathrm{Na}, \mathrm{cmol}^{-1} \mathrm{~kg}^{-1}$ & $0,067 \mathrm{~d}$ & $0,076 \mathrm{c}$ & $0,083 \mathrm{~b}$ & $0,098 \mathrm{a}$ & $0,077 \mathrm{c}$ & $0,071 \mathrm{~d}$ \\
$\mathrm{Ca} / \mathrm{SB}^{+}(\%)$ & $72,1 \mathrm{~d}$ & $73,9 \mathrm{a}$ & $73,0 \mathrm{c}$ & $73,2 \mathrm{bc}$ & $73,8 \mathrm{ab}$ & $73,8 \mathrm{ab}$ \\
\hline
\end{tabular}

${ }^{+} \mathrm{SB}$, suma de bases $(\mathrm{Ca}+\mathrm{Mg}+\mathrm{K}+\mathrm{Na})$.

Letras distintas en una misma fila indican diferencia estadística entre medias según test de Tukey $(p<0,05)$

de el inicio, respecto de otro suelo con el cual se compare, mantiene está característica en periodos cortos de evaluación como el empleado en las incubaciones de este experimento (Laos et al., 2000; Hirzel et al., 2010).

\section{Efecto del tiempo}

Para los tiempos de incubación y como promedio de ambos suelos y de los tratamientos de fertilización cálcica (Tabla 4), sólo se obtuvo diferencias en la evolución de las concentraciones de $\mathrm{Mg}$, Na y de la relación $\mathrm{Ca} / \mathrm{SB}$, como se puede observar también en las Figs. 1 y 2, con mayores variaciones para la concentración de $\mathrm{Na}$ en el suelo franco (Figs. If y 2f). Sin embargo, la interacción suelo $\mathrm{x}$ tiempo de incubación explica también diferencias en la evolución de la concentración de Ca para el suelo franco (Tabla 2 y Fig. 1c). Esta mayor variación de concentración de Ca como promedio del tiempo de incubación en el suelo franco (123,2 $\mathrm{mg} \mathrm{kg}^{-1}$ respecto de $98,8 \mathrm{mg}$ $\mathrm{kg}^{-1}$ en el suelo arcilloso, Tablas 5 y 6 y Fig. 1c y 2c) puede ser explicada por el menor contenido de arcillas de este suelo (Hossain et al., 2015), lo cual genera menor poder tampón y mayor probabilidad de cambios en la concentración de bases disponibles (Havlin et al., 1999; Fageria y Nascente, 2014). Las variables $\mathrm{pH}$ y CE fueron muy estables en el periodo de evaluación en ambos suelos (Tabla 4 y Figs. 1a, 1b, 2a y 2b), asociado al corto periodo de evaluación. Variaciones de $\mathrm{pH}$ y CE en incubaciones de suelo por periodos más largos de tiempo (8 a 16 semanas) han sido reportadas por Hirzel et al. (2010). A su vez, estos autores reportaron también variaciones en la concentración de $\mathrm{K}$ disponible, que en el presente estudio sólo se detectaron en el suelo arcilloso.

La evolución en la relación de $\mathrm{Ca} / \mathrm{SB}$ como promedio de ambos suelos y de los tratamientos de fertilización cálcica, presentó diferencias significativas durante el periodo de evaluación (Tabla 5), sin embargo estas diferencias fueron muy ajustadas (Figs. $1 \mathrm{~g}$ y 2g), y con efectos erráticos para algunos tratamientos, que fueron detectados en las interacciones obtenidas entre todas las fuentes de variación (Tabla 2). Estas variaciones erráticas pueden ser explicadas tanto por el error experimental e interacciones entre fuentes de variación, como por los procesos de intercambio catiónico del suelo, que afectan las tasas de adsorción y desorción de bases (Havlin et al. 1999; Fageria and Nascente, 2014), sobre todo cuando se emplean fertilizantes cálcicos de diferente anión acompañante (Fageria y Nascente, 2014), como ha sido reportado también por Castro y Crusciol (2013) para cambios de largo plazo en la concentración de $\mathrm{Ca}, \mathrm{Mg}$ y SB de un suelo Hapludox cuando se empleó carbonato y silicato de Ca.

Si bien en el corto periodo de evaluación del presente estudio, orientado a las variaciones en propiedades químicas a corto plazo, no se obtuvieron diferencias de evolución en la concentración y valores en algunos de los parámetros químicos analizados $(\mathrm{p}>0,05)$ como promedio de ambos suelos y de los tratamientos de fertilización cálcica (Tablas 2 y 4), llama la atención las variaciones cuantitativas en la concentración de $\mathrm{K}$ en ambos suelos. Lo anterior puede obedecer a variaciones significativas en la concentración de otras bases como Mg y Na ( $p>0,05)$ (Tabla 4, y Figs. 1d, 2d, 1f, 2f), además de Ca en el suelo franco (Fig. 1c), como efecto de procesos de intercambio de cationes en el suelo (Havlin et al. 1999; Fageria y Nascente, 2014). Al respecto, variaciones en la concentración de $\mathrm{K}$ han sido reportadas para incubaciones de un suelo volcánico por periodos más largos de tiempo (8 a 16 semanas), pero de menor magnitud debido a que en ese 
a
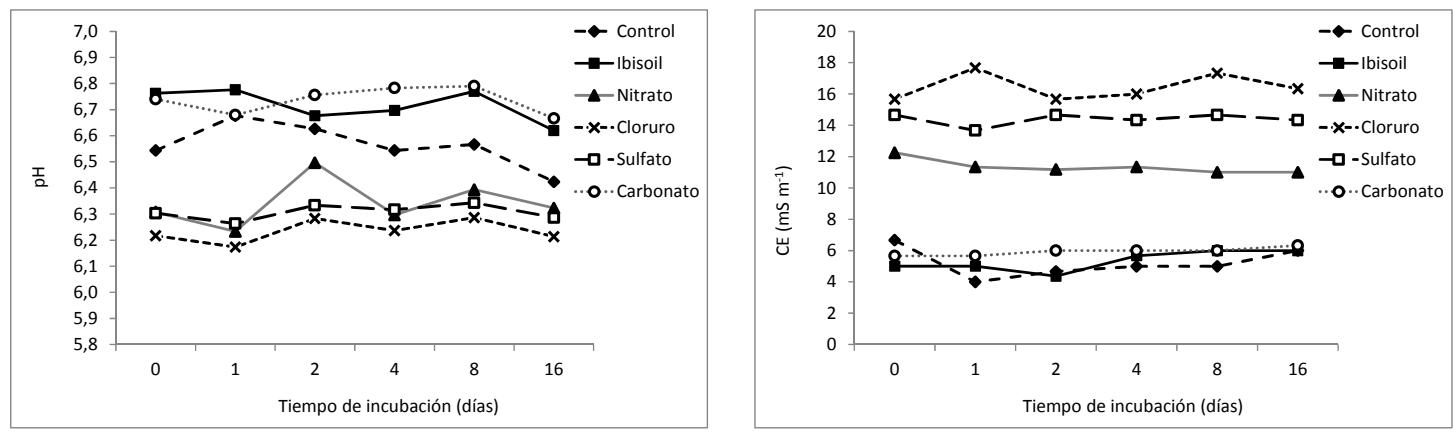

$\mathrm{c}$

$\mathrm{d}$
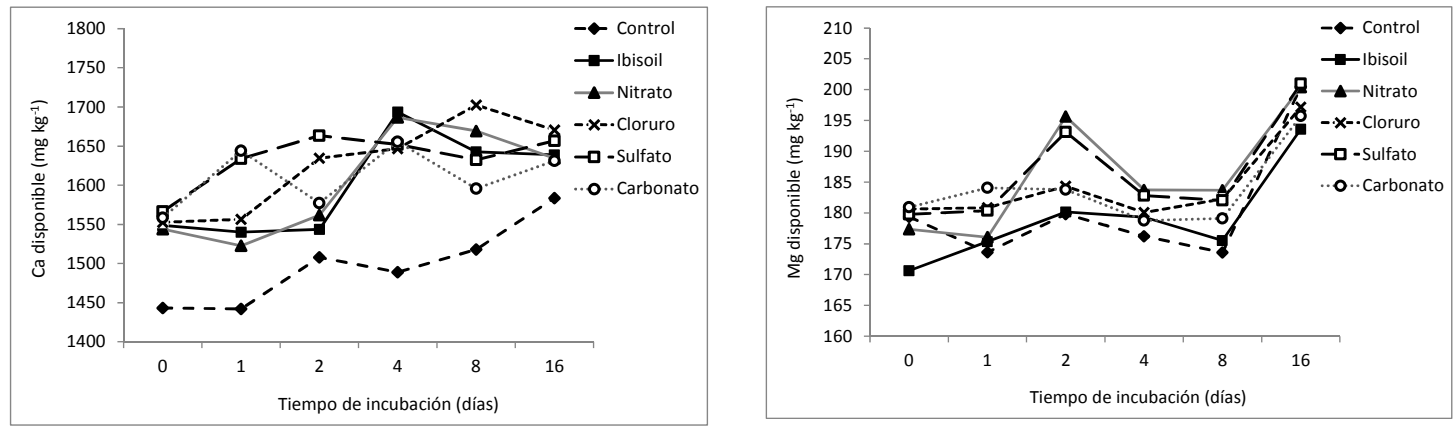

$\mathrm{e}$

$\mathrm{f}$
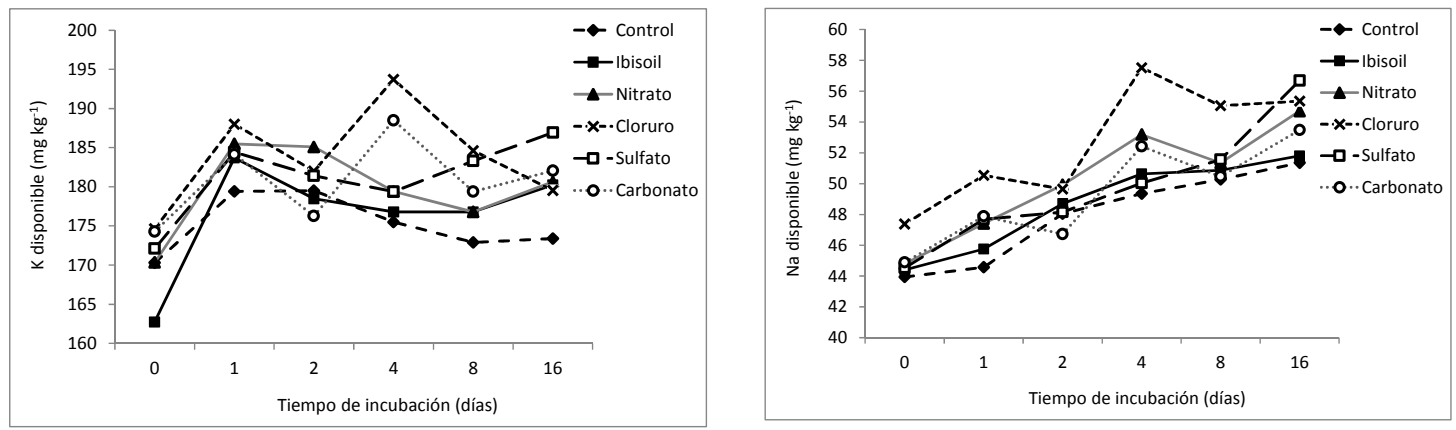

$\mathrm{g}$

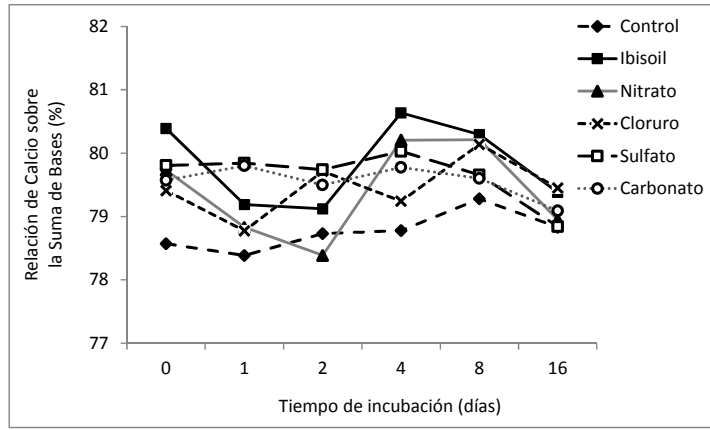

Fig. 1. Evolución de las propiedades químicas del suelo franco durante un periodo de incubación de 16 días en condiciones aeróbicas, con temperatura de $25^{\circ} \mathrm{C}$ y $80 \%$ de la humedad aprovechable, en respuesta a la aplicación de diferentes fertilizantes cálcicos: a) pH, b) CE, c) Ca disponible, d) $\mathrm{Mg}$ disponible, e) $\mathrm{K}$ disponible, f) Na disponible, g) relación de Ca sobre la suma de bases.

Fig. 1. Evolution of the chemical properties of the loam soil during an incubation period of $\mathbf{1 6}$ days under aerobic conditions, with a temperature of $25^{\circ} \mathrm{C}$ and $80 \%$ of available water, in response to the application of different calcium fertilizers: a) $\mathrm{pH}$, b) EC, c) available $\mathrm{Ca}$, d) available $\mathrm{Mg}$, e) available $\mathrm{K}$, $\mathrm{f}$ ) available $\mathrm{Na}$, g) ratio of $\mathrm{Ca}$ on the sum of bases. 
a

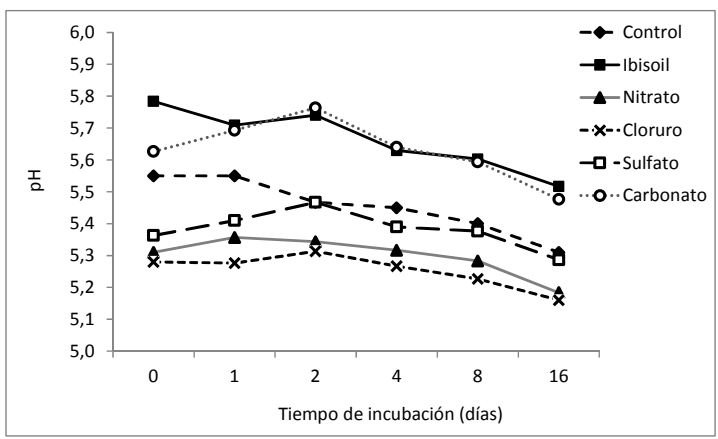

c
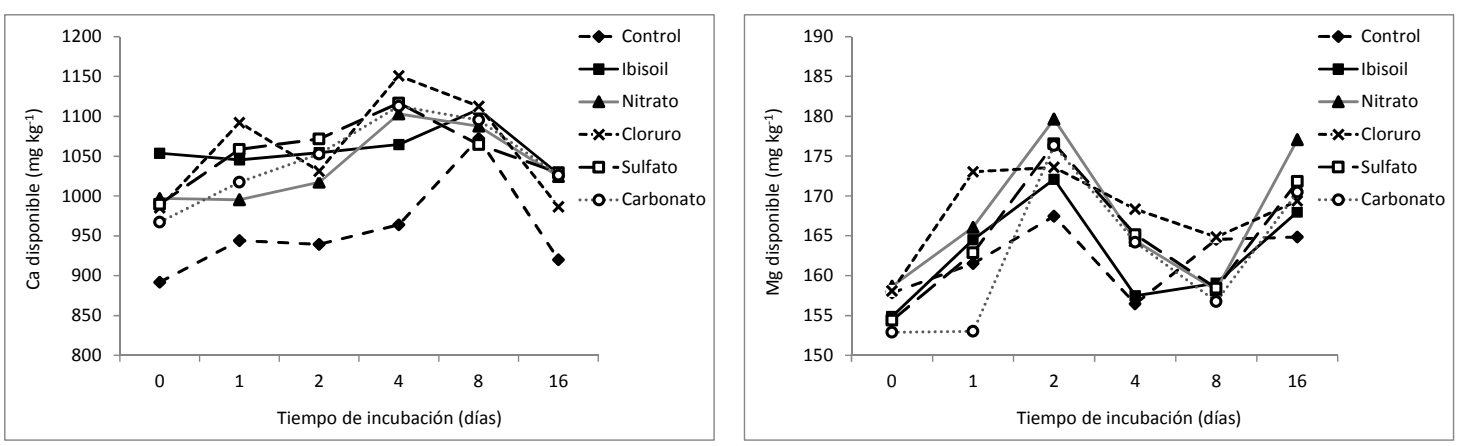

e
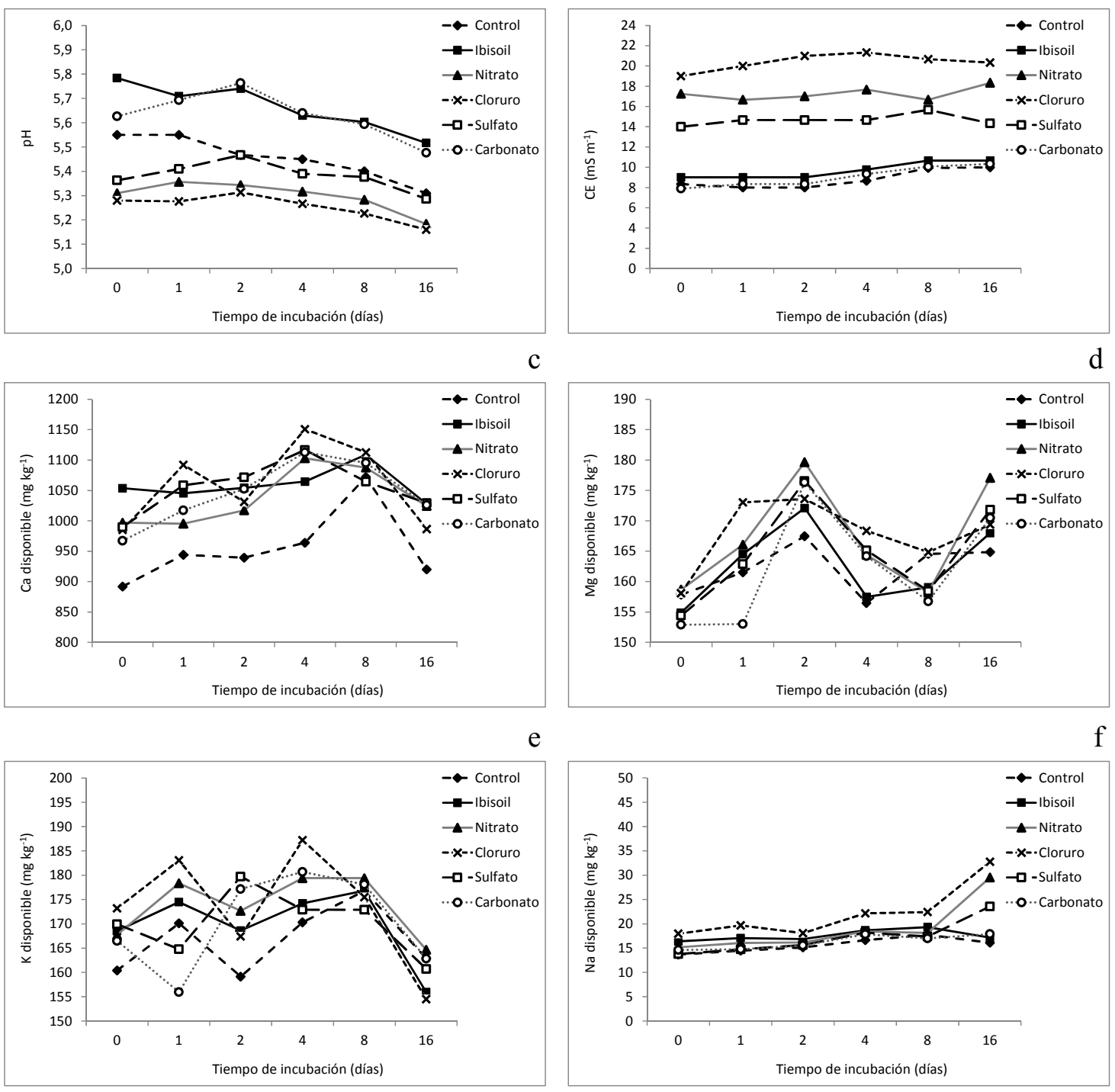

d

$\mathrm{g}$

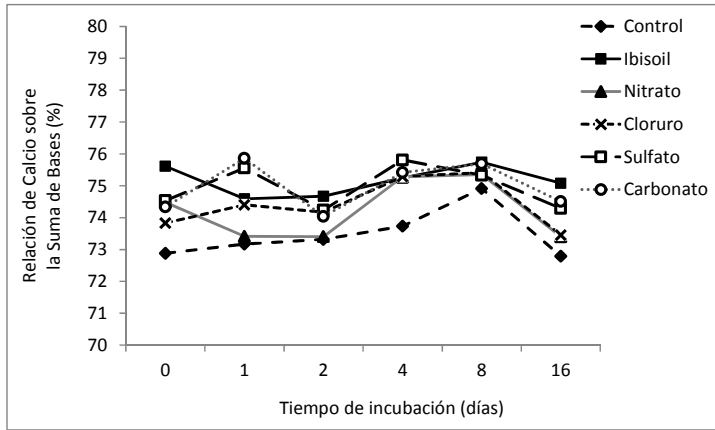

Fig. 2. Evolución de las propiedades químicas del suelo arcilloso durante un periodo de incubación de 16 días en condiciones aeróbicas, con temperatura de $25^{\circ} \mathrm{C}$ y $80 \%$ de la humedad aprovechable, en respuesta a la aplicación de diferentes fertilizantes cálcicos: a) $\mathrm{pH}, \mathrm{b}$ ) $\mathrm{CE}$, c) Ca disponible, d) $\mathrm{Mg}$ disponible, e) $\mathrm{K}$ disponible, f) Na disponible, g) relación de Ca sobre la suma de bases.

Fig. 2. Evolution of the chemical properties of the clay soil during an incubation period of 16 days under aerobic conditions, with a temperature of $25^{\circ} \mathrm{C}$ and $80 \%$ of available water, in response to the application of different calcium fertilizers: a) $\mathrm{pH}, \mathrm{b}$ ) $\mathrm{EC}, \mathrm{c}$ ) available $\mathrm{Ca}$, d) available $\mathrm{Mg}$, e) available $\mathrm{K}$, f) available $\mathrm{Na}, \mathrm{g}$ ) ratio of $\mathrm{Ca}$ on the sum of bases. 
caso no se agregaron enmiendas cálcicas (Hirzel et al., 2010).

\section{Efecto de cada fertilizante cálcico}

Respecto a los efectos de cada fertilizante cálcico como promedio del periodo de evaluación en cada suelo, en el suelo franco (Tabla 5) se obtuvieron diferencias en todas las propiedades químicas analizadas, excepto en la concentración de K. En tanto, en el suelo arcilloso estas diferencias fueron detectadas en todas las propiedades excepto en el $\mathrm{pH}$ (Tabla 6). Estas diferencias entre suelos y sus interacciones con la mayoría de las variables químicas analizadas fueron también detectadas en el análisis de significancia (Tabla 2).

\section{Efecto de cada fertilizante cálcico en el suelo franco.}

En el suelo franco el mayor $\mathrm{pH}$ se logró con la aplicación de carbonato de $\mathrm{Ca}(\mathrm{p}<0,05)($ Tabla $5)$, situación esperable y reportada también por otros investigadores (Castro and Crusciol, 2013; Fageria y Nascente; 2014; Fageria et al., 2014; Moreira et al., 2015). Luego y en orden descendente, el mayor pH se logró con Ibisoil, Control, Nitrato, Sulfato y Cloruro de $\mathrm{Ca}$, todos diferentes entre sí $(p<0,05)$ (Tabla 5). Un efecto similar fue obtenido en la evolución del $\mathrm{pH}$ durante todo el periodo de evaluación (Fig. 1a). El menor $\mathrm{pH}$ o efecto de acidificación logrado con el uso de Nitrato, Sulfato y Cloruro de Ca respecto al control, se puede explicar por el efecto estimulante de la mineralización del suelo y producción de ácidos orgánicos cuando se emplea N y $\mathrm{S}$ en el caso de Nitrato y Sulfato de Ca (Fageria y Nascente, 2014), o S, sin embargo se habría esperado un efecto nulo en el $\mathrm{pH}$ con el uso de Cloruro de Ca en la baja concentración empleada. Al respecto se ha descrito que el estrés salino en el suelo ( $\mathrm{Cl}$ o $\mathrm{Na}$ ) estimula mecanismos de tolerancia en las poblaciones microbianas del suelo, sintetizando compuestos orgánicos que aminoran las diferencias de potencial entre el suelo y las células de dichos organismos, aumentando la excreción de compuestos orgánicos al medio (De Souza y Francisconi, 2012), que podrían aumentar la mineralización y con ello la reducción del pH (Frageria y Nascente, 2014).

La CE fue mayor con el Cloruro de Ca $(\mathrm{p}<0,05)$ (Tabla 5), seguida del Sulfato, que a su vez fue mayor al Nitrato $(p<0,05)$, y todos ellos mayores al Ibisoil, Carbonato de Ca y al control, que fueron similares entre sí $(p>0,05)$. El mismo efecto fue obtenido en la evolución da la CE durante todo el periodo de evaluación (Fig. 1a). Al respecto se ha reportado el mayor efecto sobre la CE con el uso de algunas sales como Cloruro, Sulfato y Nitrato, por su efecto iónico y de alteración de potenciales hídricos a nivel de suelo (Bar-Yosef, 1999).
Respecto a la concentración de Ca como promedio del periodo de evaluación, todos los tratamientos superaron al control $(p<0,05)$, sin diferencias entre ellos $(p>0,05)$ (Tabla 5). La ausencia de diferencia entre la concentración de Ca obtenida como promedio del periodo puede atribuirse a la dosis de Ca empleada, que fue la misma con todos los productos. Otra causa es la preparación de los fertilizantes previo a su aplicación, dado que la molienda de los fertilizantes sólidos de menor solubilidad (Carbonato y Sulfato de Ca) mejoró la solubilidad en agua y el aporte de Ca disponible al suelo. Cabe destacar que en la evolución parcial de la concentración de Ca se observa un mayor incremento de disponibilidad después de la aplicación de los tratamientos con el uso de Sulfato y Carbonato (Fig. 1c), situación que pudo estar afectada por la preparación del fertilizante previo a su aplicación (molienda y aumento de superficie de contacto y solubilidad), lo que no representa la realidad a nivel de campo. Incrementos de $\mathrm{Ca}$ en el control en ambos suelos (Fig. 1c y 2c) son derivados del cambio en el pH del suelo (Fig. 1a y 2a) como efecto de una mayor desorción de cationes frente a una reducción del $\mathrm{pH}$ a través del periodo de incubación de suelos (Havlin et al. 1999; Fageria y Nascente, 2014).

La concentración de $\mathrm{Mg}$ como promedio del periodo de evaluación fue mayor con el uso de Sulfato de Ca, que sólo superó a Ibisoil $(\mathrm{p}<0,05)$ (Tabla 5). La presencia de ácidos carboxílicos en Ibisoil pudo haber generado complejos con el $\mathrm{Mg}$ a nivel de suelo, y haber reducido su disponibilidad por efecto de quelatación (Havlin et al. 1999). En el caso de la concentración de Na como promedio del periodo de evaluación (Tabla 5), la mayor concentración se obtuvo con el uso de Cloruro de $\mathrm{Ca}$, que superó a los tratamientos Control, Ibisoil, Sulfato y Carbonato. Este efecto se observó también en la evolución de la concentración de $\mathrm{Na}$ durante todo el periodo de evaluación (Fig. 1f). Al respecto, la aplicación de Cloro en el suelo presenta afinidad química con el $\mathrm{Na}$, pudiendo incrementar su disponibilidad, y a su vez también la CE (Bar-Yosef, 1999), como ocurrió en este suelo (Tabla 5 y Fig. 1b). Respecto a la relación de Ca sobre la SB como promedio del periodo, los mayores valores se obtuvieron con el uso de Ibisoil y Sulfato, que sólo superaron al uso de Nitrato $y$ al control $(\mathrm{p}<0,05)$. A su vez, los tratamientos con Nitrato, Cloruro y Carbonato, superaron al control sin fertilización cálcica $(\mathrm{p}<0,05)$ (Tabla 5). La evolución parcial de esta relación muestra un mayor efecto del Ibisoil al momento de realizar la aplicación (Fig. 1g), asociado probablemente a su formulación líquida y mayor solubilidad, efecto que también se habría esperado con el uso de Nitrato y Cloruro de Ca. 


\section{Efecto de cada fertilizante cálcico en el suelo arcilloso}

En el suelo arcilloso (Tabla 6) la mayor CE fue obtenida con el Cloruro de $\mathrm{Ca}(\mathrm{p}<0,05)$ (Tabla 5), seguida del Nitrato, que a su vez fue mayor al Sulfato $(p<0,05)$, y todos ellos mayores al Ibisoil Ca y Carbonato de Ca $(\mathrm{p}<0,05)$; a su vez el valor obtenido con el Carbonato fue similar al Control $(p>0,05)$. El mismo efecto fue obtenido en la evolución de la CE durante todo el periodo de evaluación (Fig. 2a). Estos efectos ya fueron reportados para el suelo franco (Bar-Yosef, 1999). Respecto a la concentración de $\mathrm{Ca}$, al igual que en el suelo franco, todos los tratamientos superaron al control $(\mathrm{p}<0,05)$, sin diferencias entre ellos ( $p$ $>0,05$ ) (Tabla 6). Esta ausencia de diferencia entre los fertilizantes cálcicos fue discutida en el suelo franco. La evolución parcial de la concentración de Ca muestra una mayor disponibilidad inicial de Ca con el uso de Ibisoil, que luego se asemeja a los otros tratamientos de fertilización cálcica, con efectos erráticos de cada tratamiento durante el periodo de evaluación (Fig. 2c) lo que se atribuye al error experimental y a las interacciones obtenidas entre las fuentes de variación.

La concentración de $\mathrm{Mg}$ como promedio del periodo de evaluación fue mayor con el uso de Cloruro de Ca, que sólo superó al Control y al uso de Carbonato de $\mathrm{Ca}(\mathrm{p}<0,05)$, sin diferencia entre ellos ( $p>0,05$ ) (Tabla 6). Por su parte, la concentración de $\mathrm{K}$ fue mayor con el uso de Nitrato y Cloruro de $\mathrm{Ca}(\mathrm{p}<0,05)$, que sólo superaron al control sin fertilización (Tabla 6). Estas diferencias pueden ser explicadas por incrementos en la desorción de bases desde la CIC (Havlin et al., 1999; Fageria y Nascente, 2014), que también se vio reflejado en una mayor CE con estos dos tratamientos (Tabla 6). Este efecto observado en ambos suelos (Fig. 1 y 2), fue mayor en el suelo franco (Fig. 1) debido al menor poder tampón presente en un suelo con menor contenido de arcillas (Havlin et al., 1999).

Para la concentración de $\mathrm{Na}$ (Tabla 6), y al igual que en el suelo franco, la mayor concentración se obtuvo con el uso de Cloruro de $\mathrm{Ca}(\mathrm{p}<0,05)$. A su vez, los tratamientos Carbonato y Nitrato de Ca superaron a los tratamientos Ibisoil y Sulfato, sin diferencias entre estos dos tratamientos $(p>0,05)$. Este efecto se observó también en la evolución de la concentración de Na durante todo el periodo de evaluación (Fig. 2f), que se discutió para el suelo franco. Respecto a la relación de Ca sobre la SB como promedio del periodo, los mayores valores se obtuvieron con el uso de Ibisoil, Sulfato y Carbonato de Ca (Tabla 6), superando Ibisoil a los otros tratamientos $(p<0,05)$. Por su parte los tratamientos Sulfato y Carbonato de Ca superaron al Nitrato de Ca y este tratamientos a su vez superó al control $(p<0,05)$ (Tabla 6). La evolución parcial de esta relación muestra un mayor efecto inicial del Ibisoil (Fig. 2g), efecto anteriormente explicado en los resultados obtenidos para el suelo franco.

Finalmente, se sugiere la evaluación de fuentes cálcicas con plantas indicadoras, dado que los iones acompañantes al Ca pueden generar efectos en la biomasa vegetal no detectables en experimentos con ausencia de plantas (Román y Gutierrez, 1998).

\section{CONCLUSIONES}

La aplicación de diferentes fuentes cálcicas como polvo fino o en formulación líquida en los dos suelos evaluados en este estudio, generó cambios de corto plazo en algunas de las propiedades químicas analizadas. Estos cambios fueron también afectados por el tiempo de evaluación, con diferencias en las magnitudes de variación para cada tiempo y tipo de suelo dentro del periodo de 16 días de evaluación.

En el suelo franco, la concentración de Ca y K disponible no fue afectada por las fuentes cálcicas empleadas, en tanto que para el Ca hubo diferencias sólo respecto al control. A su vez, con el uso de Carbonato de Ca se logró el mayor $\mathrm{pH}$. La mayor concentración de $\mathrm{Mg}$ se logró con el uso de Sulfato de Ca. La aplicación de Ibisoil y Sulfato de Ca permitieron obtener los mayores valores de relación de disponibilidad de $\mathrm{Ca} / \mathrm{SB}$.

En el suelo arcilloso, el uso de diferentes fuentes cálcicas no generó diferencias en el $\mathrm{pH}$ y concentraciones de Ca y K disponible, manifestando diferencias sólo respecto al control. La mayor concentración de $\mathrm{Mg}$ se logró con el uso de Cloruro de Ca. La aplicación de Ibisoil permitió obtener los mayores valores de relación de disponibilidad de $\mathrm{Ca} / \mathrm{SB}$.

En ambos suelos, la menor CE se obtuvo con el uso de Ibisoil y Carbonato de Ca. Por su parte, la mayor concentración de Na y CE se obtuvo con el uso de Cloruro de Ca. A su vez, la mayor disponibilidad inmediata de Ca medido a través de la relación $\mathrm{Ca} / \mathrm{SB}$ se logró con el uso de Ibisoil Ca.

\section{LITERATURA CITADA}

Baldi, E., M. Toselli, G. Marcolini, and B. Marangoni. 2006. Effect of mineral and organic fertilization on soil chemical, biological and physical fertility in a commercial peach orchard. Acta Horticulturae 721:55-62.

Bar-Yosef, B. 1999. Advances in Fertigation. Advances in Agronomy 65:1-77. 
Burt, R. 2004. Soil Survey Laboratory Methods Manual. Soil Survey Investigations Report No. 42. Version 4.0. 700 p. Natural Resources Conservation Service, United States Department of Agriculture, Washington DC, USA.

Caires, E.F., A. Haliski, A.R. Bini, and D.A. Scharr. 2015. Surface liming and nitrogen fertilization for crop grain production under no-till management in Brazil. Europ. J. Agronomy 66:4153.

Castro, G.S.A., and C.A.C. Crusciol. 2013. Effects of superficial liming and silicate application on soil fertility and crop yield under rotation. Geoderma 195-196:234-242.

De Souza, M., and E. Francisconi. 2012. Effect of salinity on soil microorganisms, soil health and land use management. p. 177-198. In Hernández, M. (ed.). Soil health and land use management. ISBN: 978-953-307-614-0. Available at http://www.intechopen.com/books/ soil-health-and-land-usemanagement/effect-of-salinity-on-soil-microorganisms. (Accessed 1 February 2016).

Fageria, N., and A. Nascente. 2014. Management of soil acidity of South American soils for sustainable crop production. Advances in Agronomy 128:221-275.

Fageria, N.K., A. Moreira, L.A.C. Moraes, and M.F. Moraes. 2014. Influence of lime and gypsum on yield and yield components of soybean and changes in soil chemical properties. Com. Soil Sci. Plant Anal. 45:271-283.

Flores, D.A., M.E. Vázquez, J.C. Mildemberg, y J. Beltrano. 2014. Efecto de la fertilización cálcica sobre el rendimiento y tamaño de fruto en arándano alto del sur (Vaccinium corymbosum L.). Horticultura Argentina 33(81):31-36.

Havlin, J.L., S.L. Tisdale, W. Nelson, J. Havlin, and J. Beaton. 1999. Soil fertility and fertilizers. An introduction to nutrient management. $6^{\text {th }}$ ed. 503 p. Prentice-Hall, Upper Saddle River, New Jersey, USA.

Hirzel, J., I. Matus, F. Novoa, and I. Walter. 2007. Effect of poultry litter on silage maize (Zea mays L.) production and nutrient uptake. Spain J. Agric. Res. 5(1):102-109.

Hirzel, J., P. Undurraga, and I. Walter. 2010. Mineralization of nitrogen and nutrients released in a volcanic soil amended with poultry manure. Chilean Journal of Agricultural Research 70(1):113-121.

Hossain, N., M. Muhibbullah, K. Barkat, and M. Hossan. 2015. Relationship between soil salinity and physico-chemical properties of paddy field soils of Jhilwanja Union, Cox's Bazar, Bangladesh. Journal of Agricultural Science 7:166-180.
Laos, F., P. Satti, I. Walter, M.J. Mazzarino, and S. Moyano. 2000. Nutrient availability of composted and noncomposted residues in a Patagonian Xeric Mollisol. Biol. Fertil. Soils 31:462469.

Maathuis, F.J.M. 2009. Physiological functions of mineral macronutrients. Current Opinion in Plant Biology 12:250-258.

Marschner, P. (ed.) 2012. Mineral nutrition of higher plants. 3rd ed. 651 p. Academic Press, London, UK.

Moore, J., and R. Ouimet. 2014. Effects of two types of Ca fertilizer on sugar maple nutrition, vigor and growth after 7 years. Forest Ecology and Management 320:1-5.

Moreira, A., G.J. Sfredo, L.A.C. Moraes, and N.K. Fageria. 2015. Lime and cattle manure in soil fertility and soybean grain yield cultivated in tropical soil. Com. Soil Sci. Plant Anal. 46:1157-1169.

Román, M.L.F., y M.A. Gutiérrez C. 1998. Evaluación de ácidos carboxílicos y nitrato de calcio para incrementar calidad, cantidad y vida de anaquel en tres tipos de melón. Terra 16(2):4954.

Sadzawka, A., M.A. Carrasco, R. Grez Z, M.de la L. Mora, H. Flores P, and A. Neaman. 2006. Metodos de análisis recomendados para los suelos de Chile. Revision 2006. 164 p. Serie Actas. Instituto de Investigaciones Agropecuarias, CRI La Platina, Santiago, Chile.

SAS Institute. 1989. Usage and reference. Version 6. 501 p. SAS Institute, Cary, North Carolina, USA.

Tagliavini, M., C. Zavalloni, A. Rombola, M. Quartieri, D. Malaguti, and F. Mazzanti. 2000. Mineral nutrient partitioning to fruits of deciduos trees. Acta Horticulturae 512:131-140.

USDA. 2014. Keys to Soil Taxonomy. 12th. ed. 360 p. United States Department of Agriculture (USDA), Washington DC, USA.

Zavalloni, C., B. Marangoni, M. Tagliavini, and D. Scudellari. 2001. Dynamics of uptake of calcium, potasium and magnesium into apple fruit in a high density planting. Proceedings of the Fourth International Symposium on Mineral Nutrition of Deciduous Fruit Crops. Acta Horticulturae 564:113-122. 